\title{
CONSISTENT SPHERICAL NLTE-MODELS FOR BE-STARS
}

\author{
Peter Höflich \\ Institut fuir Theoretische Astrophysik \\ Im Neuenheimer Feld 561,D-6900 Heidelberg \\ West Germany
}

\begin{abstract}
Spherical NLTE-model photospheres surrounded by envelopes are calculated in order to interpret classical Be-stars. The existence of the HII-region can be understood by such models. The dependence of the calculated spectra on parameters corresponds to the observations. Applications to special objects show good agreement between the predicted and observed spectra in the visual and inf rared wavelengh range.
\end{abstract}

\section{INTRODUCTION}

In the last years many new details have been observed for classical Bestars. Nevertheless the physical processes which cause the Be-phenomena are almost unknown. The interpretation of the H-spectrum (i.e. lines, Balmer jump and infrared excess) plays an important role, because an accurate knowledge of this region is a useful test for the processes dominating the envelope. The uncertainties are due to the difficulties for the construction of models. They are caused by the lack of knowledge concerning the geometry and by the high densities which are expected in the nebulae. Therefore one can use neither the conventional assumptions for atmospheres (i.e. LTE, plane geometry) nor those for "classical" HII-regions (i.e. occupation numbers given by the cases of Menzel and Baker, constant temperature). Furthermore, the existence of high density HII-regions around B-stars cannot be explained by ionization due to Lyman continuum photons.

\section{MODEL CONSTRUCTION}

A computer code for the construction of spherically extended NLTE models was developed (seeking to minimize approximations concerning the physical treatment, to reduce the number of free parameters (see Figure 1) and to get an unique interpretation). We consider a hydrostatic photosphere which is joined at a radius $R_{*}$ and a foot point density $N_{0}$ to a spherical shell with a density distribution $N(r) \sim r^{-n}$ and an outer radius $R_{\text {out }}$. Both the photosphere and the shell are assumed to be in radiative equilibrium. For hydrogen up to eight levels are allowed to deviate from LTE. All transitions between the NLTE-levels are included in the statistical equation as well as in the radiative transport equation which is solved by the integral methor of Schmidt-Burgk (1975). 


\section{GENERAL RESULTS}

The existence of HII-regions around B stars can be understood by photoionization due to Balmer continuum photons, strong bound-bound collisions, small radiative net rates, and small dilution factors in contrast to "classical" HII-regions.

There is a complicated interplay between densities, temperatures and occupation numbers.

The backwarming of the outer photospheric layers caused by the envelope may amount to several thousand degrees and influences the spectral features (line fluxes, Balmer and Paschen jumps, IR-colors etc.).

\section{APPLICATION TO CLASSICAL Be-STARS}

The dependence of the calculated spectra on parameters agrees well with the observed spectra.

We compare the observed and calculated spectra of a few Be-stars (HD58343, X Oph, 60 Cygni, $\epsilon$ PsA, o Aqu). In general, they show good agreement. As an example the lines of o Aquarii are shown in Figure 1.

To explain the spectra we have to choose the free parameters in the following range: The extensions of the envelopes are between 2 and 10 $R_{*}$. The density law has an exponent between 2 and 2.5 with a footpoint density $\mathrm{N}_{\mathrm{O}}$ in the range $2 \cdot 10^{11}$ to $10^{12} \mathrm{~cm}^{-3}$. The velocity fields must be chosen between 10 and $150 \mathrm{~km} / \mathrm{sec}$. For some objects (HD58343, $\chi$ Oph) the calculated line fluxes in the wings are too low. This may be explained by higher densities or higher velocities in the inner region of the nebulae, or smaller gravitational acceleration in the underlying photosphere (i.e. smaller absorption profiles).

For more information see Höflich(1986).

Figure 1: $\mathrm{H}_{\alpha}, \mathrm{H}_{\beta}$ and $\mathrm{H}_{\gamma}$ profiles (measured by Dachs et al. (1986) in Nov.1981) in comparison with the calculated ones (-) which are convolved by an instrumental profile (of a half width of $2 \AA$ for $\mathrm{H}_{\alpha}$ and $1 \AA$ for $\mathrm{H}_{\beta}$ and $\mathrm{H}_{\gamma}$ ). The model has the following free parameters:

$\mathrm{T}_{\text {eff }}=13500 \mathrm{~K} ; \mathrm{M}_{*}=3.7 \mathrm{M}_{\odot} ; \mathrm{R}_{*}=4 \mathrm{R}_{\odot} ; \mathrm{N}(\mathrm{r})=$ $2.5 \cdot 10^{11}\left(R_{*} / \mathrm{r}\right)^{2} \mathrm{~cm}^{-3} ; \mathrm{R}_{\text {out }}=14.7 \mathrm{R}_{\odot}$; $v_{\text {turb }}=90 \mathrm{~km} / \mathrm{sec}$ (in the envelope).

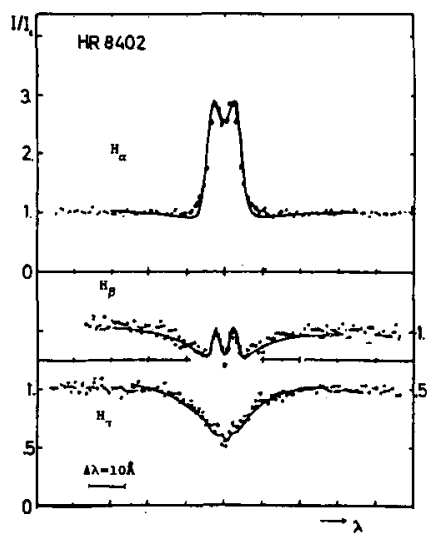

\section{REFERENCES}

Dachs, J. ; Hanuschik, R; Kai ser ,D. ; Ball lereau, D. ; Bouchet, P. ;Kiehl ing, R. ;Kozok, J. ;Rudolph,R.;Schlosser,W. 1986, Astron.Astrophys.Suppl.Ser. 63, 87 Höf lich,P. 1986, Doktorarbeit, Univ. Heidelberg Schmidt-Burgk, J. 1975. As tron. As trophys.ㄴ. 249 


\section{DISCUSSION FOLLOWING HOFLICH}

Buscombe:

In my line profile for $\mathrm{H} \alpha$ in o Andromedae the central reversal was much deeper.

Hoflich:

This is an effect of the instrumental resolution.

Bolton:

Can you reproduce the polarization observed in these stars which is usually attributed to flattening of the circumstellar envelope?

Hoflich:

No, because the model is spherically symmetric. But the polarization can be explained as well by a flattened geometry of the envelope as by a flattened geometry of the fast rotating star as Metz has shown in Munich. Therefore the polarization gives you no direct information about the envelope. I want to note additionally that the existence of the HII region around late type B-stars cannot be explained by self consistent models if you assume a flattened disc ( $\mathrm{v}_{\text {polar }}<2 \mathrm{v}_{\text {polar }}<2 \mathrm{v}_{\text {equator }}$ ).

Baade:

The difference between the observed $\mathrm{H} \alpha$ profile of And and your model profile is largest in the wings. Dr. Brown has just told us that her model reproduces such wings only if she assumes a very flat dish. Do you see a possibility within your model to obtain broad wings in a different way?

Hoflich:

Yes; it is possible by Stark broadening and Thomson scattering over a line. In this special case the differences are due to the fact that we have assumed $\log (\mathrm{g})=4.0$ but the star seems to have a slightly lower $\log (\mathrm{g})$, as can be seen in the absorption wings of $\mathrm{H} \alpha$. So the difference is due to a too broad photospheric absorption line and not to low emission in the shell. 